\title{
Why Breast Cancer Risk Models Fail: The Case of Lobular Carcinoma In Situ
}

\author{
David M. Euhus, MD \\ Johns Hopkins University School of Medicine, Baltimore, MD
}

The first photomicrograph demonstrating lobular acini distended with bland, discohesive cells was published in 1908. ${ }^{1}$ The condition was named lobular carcinoma in situ (LCIS) in $1941,{ }^{2}$ however, by 1971, Haagensen et al. had recognized that "Lobular carcinoma in situ is a misleading and unfortunate name for this benign, noninfiltrating, special microscopical form of lobular proliferation of the mammary epithelium". ${ }^{3}$ For a long time, it was popular to teach that LCIS is a marker of risk for cancer in either breast, and not a precursor of invasive carcinoma. This is partially true. The fact is, women diagnosed with LCIS disproportionately develop invasive lobular carcinoma in the originally affected breast. The frequent occurrence of LCIS in association with invasive lobular carcinoma suggests it may be a precursor lesion. Indeed, it was recently demonstrated that LCIS is clonally related to associated ductal carcinoma in situ (DCIS) and invasive lobular carcinoma in $42 \%$ of synchronous cases. ${ }^{4}$ LCIS is a nonobligate precursor of invasive lobular carcinoma and a marker of a breast microenvironment that is permissive for the outgrowth of invasive breast cancers. Invasive cancer risk is much lower for LCIS than for untreated DCIS. The subsequent cancers are frequently multifocal/multicentric, and may develop in other quadrants or even in the opposite breast. For these reasons, LCIS is not treated surgically.

Absolute invasive breast cancer risk for LCIS is $1.1-2.0 \%$ per year, and remains stable for up to 20 years. $^{3,5-9}$ I usually quote new LCIS patients $1.5 \%$ per year, translating that into a $15 \%$ risk in 10 years and a $30 \%$

(C) Society of Surgical Oncology 2019

First Received: 10 August 2019; Published Online: 27 September 2019

D. M. Euhus, MD

e-mail: deuhus1@jhmi.edu risk in 20 years. Any woman with LCIS and 15 years of life remaining will meet the $20 \%$ lifetime risk threshold for screening magnetic resonance imaging (MRI). Given the enrichment for invasive lobular carcinomas that are difficult to detect by mammography, screening MRI is an excellent option for these women. In addition, LCIS is an estrogen-driven phenomenon, with nearly $100 \%$ of cells expressing estrogen receptor. Chemoprevention with tamoxifen has been shown to reduce breast cancer risk by $56 \%$ in these women. ${ }^{10}$ The vast majority of women aged 35 years or over with LCIS meet short- and long-term risk thresholds for chemoprevention and screening MRI. Is there really any need to further stratify the risk associated with LCIS? The fact is, most women diagnosed with LCIS never develop breast cancer. An LCIS model capable of accurately classifying women as low or very high risk would have value for focusing risk management resources where they would do the most good.

The study by Valero et al. in this issue of Annals of Surgical Oncology suggests that the Tyrer-Cuzick (TC) model is not very good at risk-stratifying LCIS patients. ${ }^{11}$ This well designed and executed study, which included 1192 women with an initial diagnosis of LCIS who did not receive chemoprevention or risk-reducing mastectomy, recorded a $1.4 \%$ per year risk for invasive cancer. The investigators assessed calibration and discrimination of the TC model for predicting subsequent invasive breast cancer. Calibration is a simple ratio between the observed and expected number of breast cancers for quantiles of calculated risk. Valero et al. found that the observed number of breast cancers was about the same across every decile of calculated risk, suggesting that the additional risk factors included in the TC model do not modify risk associated with LCIS. Discrimination is the probability that a woman 
who developed breast cancer would have a greater TC risk than a woman who never developed breast cancer. This value was 0.493 , essentially the same as the flip of a coin.

The poor performance of the TC model for risk-stratifying LCIS patients is not unexpected given the nature of LCIS as a biological entity, and way the TC model is designed. The TC model is a robust family history model that initially estimates absolute invasive breast cancer risk based on the probability that a woman carries a mutation in BRCA1, BRCA2, or an unknown predisposition gene. ${ }^{12}$ It then multiplies this risk based on the published relative risks for other risk factors. It assumes that all of these other risk factors are independent and can be simply multiplied together. This is simply not the case. When Gail et al. developed their model (which is not a robust family history model), they carefully corrected for the way that certain risk factors interacted with one another. ${ }^{13}$ For example, they found that the number of biopsies needed to be adjusted for age, and age at first live birth, needed to be adjusted for the number of first-degree relatives with breast cancer. The computationally naïve assume that $\mathrm{TC}$ is the best risk model because it incorporates the greatest number of risk factors. At issue is how it incorporates these risk factors.

In 2010, Boughey et al. reported that the TC model overestimates the risk for women with atypical hyperplasia. ${ }^{14}$ The TC developers responded to this by updating the relative risks applied to atypical hyperplasia in TC version 7. Without knowing and accounting for how other risk factors modify the risk associated with atypical hyperplasia, this is likely to be insufficient. Valero et al. used TC version 7 for their analysis, which applies an eightfold increase in risk for LCIS. ${ }^{7}$ It is unlikely that adjusting this figure up or down will solve the calibration issue. The question is, what are the factors that modify risk in LCIS patients?

Some studies find evidence that a family history of breast cancer increases the risk for LCIS patients (although only perhaps for younger patients), ${ }^{3,6}$ while others have reported that family history does not modify this risk. ${ }^{5,7,8}$ The best evidence favors that a family history of breast cancer and a history of LCIS are not multiplicative risk factors (i.e. the risks estimated for each independently cannot be simply multiplied together when both occur simultaneously). Similarly, age at diagnosis of LCIS has been found to modify risk by some $^{6}$ but not others. ${ }^{5}$ Finally, neither extent of LCIS, ${ }^{6,8}$ nor mammographic density $^{5}$ is convincingly recognized as a modifier of the risk associated with LCIS. There are currently no factors (other than chemoprevention) convincingly shown to modify the $\sim 1.5 \%$ per year breast cancer risk observed after the diagnosis of LCIS. It is very unlikely that any new weighting scheme applied to any combination of variables derived from the currently recognized collection of breast cancer risk factors will provide clinically useful risk stratification for LCIS patients. The reason for this is found in the biology of breast carcinogenesis.

Tomasetti and Vogelstein have recently proposed that variation in cancer risk between different tissue types can be explained by variation in stem cell division rates. ${ }^{15}$ This is because DNA errors are introduced during cell division. The more times a cell divides, the more likely it is to acquire a mutation capable of driving malignant transformation. Theoretically, accurate cancer risk prediction models can be constructed by accounting for environmental exposures that increase mutation rates (e.g. smoking for lung cancer) and genetic factors that interfere with DNA repair (e.g. BRCA gene mutation for a variety of cancers). Tomasetti and Vogelstein make the point that environmental factors are really only important for organs that are regularly exposed to the external environment (e.g. lung and esophagus) and that genetic predisposition is uncommon for any site. ${ }^{16}$ For most tissues, including breast, the main driver of carcinogenesis is random errors in DNA replication. This poses a problem for risk prediction and explains why 0.75 (e.g. $25 \%$ better than chance) seems to be an immovable ceiling for model discrimination.

Most of the recognized breast cancer risk factors are markers of endogenous and exogenous sex hormone exposure, and, as such, surrogates for breast epithelial cell proliferation. Benign breast disease, especially high-risk preneoplasia, tells us that the proliferating cell population has grown large enough to be detected clinically. Family history components, including polygenetic risk scores, are likely telling us something about the capacity to repair DNA changes that occur in these proliferating cells. One way to think about breast cancer risk models is that they estimate the size of the proliferating cell population. This is related to breast cancer risk but is not quite the same thing. The proliferating cells churn along, waiting for a random event that may or may not ever occur. In the case of LCIS, this event seems to occur at the rate of about $1.5 \%$ per year.

It is remarkable that breast cancer risk models perform as well as they do. Most are fairly well calibrated (i.e. they accurately sort women into low-, intermediate-, and higherrisk groups), but all run up against the discrimination barrier imposed by biology. There is no one best model. Models validate best in populations that are most like the population used to generate the model. The way forward would be to discover that 'random' replicative errors are not really random, that there are tissue or genetic factors that greatly increase or decrease the probability of these events. However, for now, $1.5 \%$ per year will need to suffice for LCIS patients. 
DISCLOSURES David M. Euhus has the following disclosures to declare: Royalties for CancerGene Connect, a web-based cancer genetics platform that incorporates risk models; and Copyright for the desktop application CancerGene that incorporates risk models

\section{REFERENCES}

1. Cornil V. Les Tumeurs du Sein. Paris: Librairie Germer Bailliere \& Co.; 1908.

2. Foote FW, Stewart FW. Lobular carcinoma in situ: a rare form of mammary cancer. Am J Pathol. 1941;17(4):491-6.

3. Haagensen CD, Lane N, Lattes R, Bodian C. Lobular neoplasia (so-called lobular carcinoma in situ) of the breast. Cancer. 1978;42(2):737-69.

4. Lee JY, Schizas M, Geyer FC, et al. Lobular carcinomas in situ display intralesion genetic heterogeneity and clonal evolution in the progression to invasive lobular carcinoma. Clin Cancer Res. 2019;25(2):674-86.

5. King TA, Pilewskie M, Muhsen S, et al. Lobular carcinoma in experience evaluating clinicopathologic features and breast cancer risk. J Clin Oncol. 2015;33(33):3945-52.

6. Bodian CA, Perzin KH, Lattes R. Lobular neoplasia. Long term risk of breast cancer and relation to other factors. Cancer. 1996;78(5):1024-34.

7. Page DL, Kidd TE Jr, Dupont WD, Simpson JF, Rogers LW. Lobular neoplasia of the breast: higher risk for subsequent invasive cancer predicted by more extensive disease. Hum Pathol. 1991;22(12):1232-39.

8. Rosen PP, Kosloff C, Lieberman PH, Adair F, Braun DW Jr. Lobular carcinoma in situ of the breast. Detailed analysis of 99 patients with average follow-up of 24 years. Am J Surg Pathol. 1978;2(3):225-51.
9. McDivitt RW, Hutter RV, Foote FW Jr, Stewart FW. In situ lobular carcinoma. A prospective follow-up study indicating cumulative patient risks. JAMA. 1967;201(2):82-6.

10. Fisher B, Costantino JP, Wickerham DL, et al. Tamoxifen for prevention of breast cancer: report of the National Surgical Adjuvant Breast and Bowel Project P-1 Study. J Natl Cancer Inst. 1998;90(18):1371-88.

11. Valero MG, Zabor EC, Park A, et al. The Tyrer-Cuzick model inaccurately predicts invasive breast cancer risk in women with LCIS. Ann Surg Oncol. 2019. https://doi.org/10.1245/s10434-01 9-07814-w.

12. Anderson H, Bladstrom A, Olsson H, Moller TR. Familial breast and ovarian cancer: a Swedish population-based register study. Am J Epidemiol. 2000;152(12):1154-63.

13. Gail MH, Brinton LA, Byar DP, et al. Projecting individualized probabilities of developing breast cancer for white females who are being examined annually. $J$ Natl Cancer Inst. 1989;81(24):1879-86.

14. Boughey JC, Hartmann LC, Anderson SS, et al. Evaluation of the Tyrer-Cuzick (International Breast Cancer Intervention Study) model for breast cancer risk prediction in women with atypical hyperplasia. J Clin Oncol. 2010;28(22):3591-96.

15. Tomasetti C, Vogelstein B. Cancer etiology. Variation in cancer risk among tissues can be explained by the number of stem cell divisions. Science. 2015;347(6217):78-81.

16. Tomasetti C, Li L, Vogelstein B. Stem cell divisions, somatic mutations, cancer etiology, and cancer prevention. Science. 2017;355(6331):1330-34.

Publisher's Note Springer Nature remains neutral with regard to jurisdictional claims in published maps and institutional affiliations. 